\title{
Retornos salariales para Colombia: un análisis cuantílico
}

\author{
Wage Returns to Colombia: a Quantile \\ Analysis
}

\section{Retornos salariais para Colômbia: uma análise cuantílico}

\author{
Cristian Darío Castillo Robayo \\ Julimar Da Silva Bichara \\ Manuel Pérez-Trujillo
}

* Estudiante de Doctorado en Economía y Empresa en la Universidad Autónoma de Madrid. Madrid, España. cristiancastillo1711@gmail.com (iD http://orcid.org/0000-0001-7569-1860

** Profesor contratado, doctor Departamento de Estructura Económica y Economía del Desarrollo Universidad Autónoma de Madrid. Madrid, España. julimar.dasilva@uam.es

*** Profesor Departamento de Economía Universidad Católica del Norte. Antofagasta, Chile. manuel.perez@ucn.cl 


\section{Resumen}

Este artículo analiza los retornos salariales de los jóvenes y adultos, comparando la influencia de la educación y la experiencia. Se examina principalmente la teoría del capital humano y las críticas a esta, a través del estudio de la relación entre educación, experiencia y salarios. El análisis empírico se basa en el modelo de Mincer, pero mediante las regresiones cuantílicas, para determinar por 10 cuantiles en cada grupo, cómo se dan los retornos salariales. Se comprueba que para los jóvenes hay mayor impacto de adquirir experiencia que la educación sobre los retornos salariales, lo que afecta la inserción laboral y el obtener mayores ingresos laborales.

Palabras clave: retorno salarial, capital humano, regresión cuantilica, educación, experiencia.

Clasificación JEL: E24, C35, I0, J01

\section{Abstract}

This article examines the wage returns of young people and adults, considering the effect of education and experience. It is taken into account, especially, the point of view of human capital theory and its criticisms, to evaluate the relationship between education, experience and salary. The empirical analysis is based on the Mincer model, but through quantile regressions, to determine by 10 quantiles in each group as wage returns are given. It is verified that for young people there is greater impact of acquire experience than education on the wage returns, which affects labor insertion and obtaining higher income.

Keywords: wage return, human capital, quantile regression, education, experience. 


\section{Resumo}

Este artigo analisa os retornos salariais dos jovens e adultos, considerando o efeito da educação e da experiência. Considera-se, especialmente, o ponto de vista da teoria de capital humano e suas críticas, para avaliar a relação entre educação, experiência e salário. A análise empírica é baseada no modelo de Mincer, através de regressões quantílicas, para estimar como são determinados os retornos salariais do quintil $10^{\circ}$. Verifica-se que a experiência produz maior impacto nos retornos salariais dos jovens que a educação, o que afeta, por tanto, a inserção laboral e a renda desse grupo de análise.

Palavras-chave: retorno salário, capital humano, de regressão quantílica, educação, experiência. 


\section{INTRODUCCIÓN}

La configuración y determinación del salario es una problemática que se ha estudiado ampliamente, ya que se puede considerar como el más importante de los precios de mercado. La valoración del factor trabajo supone no solo el intento de analizar los posibles equilibrios en el mercado de trabajo o los problemas de su manejo institucional, sino también tener en cuenta que es el valor con el que las familias cuentan para consumir los bienes y servicios que necesitan para su subsistencia y desarrollo personal y social. El salario es, por tanto, un aspecto esencial a la hora de determinar el bienestar en una sociedad moderna.

De todas las formas como se han estudiado los determinantes de los salarios en la literatura económica, la relación entre educación y mercado laboral es de las más defendidas y analizadas. Dentro de estos estudios, la teoría del capital humano ha sido ampliamente aceptada y criticada en torno a sus argumentos de cualificación y generación de productividad de las personas más educadas; pero, dentro de esta teoría, y otras más, la experiencia es un aspecto que no se ha estudiado de la misma forma. Vale decir que se hace referencia a la formación de capital humano, como la acumulación de niveles más altos de educación y experiencia; sin embargo, existe la creencia de que la educación es lo que más genera un aumento en la productividad y, por ende, en el salario de las personas.

En el caso de Colombia se puede observar que muchas empresas, más allá de los requerimientos de educación, exigen experiencia, incluso en los más jóvenes, algo que ha generado problemas en la inserción laboral de estos y en la obtención de salarios adecuados a su formación, dado que estos salarios están por debajo, en muchos casos, de lo que debiera suponer su nivel educativo. 
Por tanto, ha de evaluarse qué tanto influye en la actualidad la escolaridad de las personas y la acumulación de experiencia en la obtención de salarios.

Basándose en el modelo de Mincer de capital humano, se observaron los retornos de la educación y experiencia sobre el ingreso laboral, mediante un análisis por regresión cuantílica. Además, se compara la situación de jóvenes y adultos para ver cómo se comportan la educación y experiencia en estos grupos.

De este modo, se analizarán las diferencias actuales entre estos aspectos que conforman el capital humano y su relación con la problemática actual de bajos salarios de los jóvenes. Con lo anterior se pretende mostrar que son necesarias políticas dirigidas no solo a aumentar el nivel educativo de la población, sino además que permitan a las personas, y más que todo a los más jóvenes, acumular experiencia que complemente sus cualificaciones para superar la dificultad de los bajos salarios.

Igualmente, se observará la relación del ingreso con otras variables como la rama de actividad económica en la que laboran, contrato de trabajo y el área geográfica donde desempeñan el trabajo, para determinar cómo esto incide en los ingresos laborales. Por último, este estudio aporta un análisis del panorama actual de la cualificación para el mercado laboral, enfatizando en los jóvenes -algo que no se ha analizado ampliamente por medio de las regresiones cuantílicas en Colombia-, para mostrar que la experiencia reporta mayores retornos que la educación y es un aspecto más importante en la determinación salarial en la actualidad.

Con este propósito, este artículo se ha organizado en cinco secciones, donde se analizan los principales aportes teóricos a la formación de salarios, la teoría del capital humano y sus principales críticas, para determinar la relación entre educación y salarios, así como las referencias a la acumulación de experiencia, el análisis empírico y los principales resultados, con énfasis en el comportamiento de la educación y la experiencia. En último término, se darán las conclusiones y recomendaciones con base en los resultados.

\section{FORMACIÓN DE LOS SALARIOS}

\section{Enfoque neoclásico}

En el modelo económico neoclásico, el mercado laboral se ve como cualquier otro mercado, por tanto, busca determinar los salarios de acuerdo con las leyes de oferta y demanda; supone que los agentes económicos son racionales, es decir, que usan toda la información para maximizar sus beneficios o utilidades. Igualmente, el mercado es de competencia perfecta, hay información perfecta de las condiciones del mercado, los intercambios solo se realizan conociendo el precio (salario) en el cual el mercado se vacía y finalmente los agentes tienen expectativas racionales.

Se supone que los trabajadores poseen un nivel similar de cualificación suficiente 
para acceder a cualquier puesto de trabajo. Los requerimientos de cualificación de los puestos de trabajo en el sistema productivo son similares y por último se da la flexibilización de los salarios. El suponer información perfecta permite ver que el coste de la movilidad externa es nulo, por lo tanto, hay movilidad de trabajadores hacia los sectores más productivos.

Además, al suponer rendimientos decrecientes de escala, una disminución en el nivel de empleo hace que la productividad marginal del trabajo aumente, lo que provocaun desplazamiento de la curva de demanda, lo cual, a su vez, ocasiona que se requiera más fuerza de trabajo (Zuluaga \& Manzur, 2012). Los desajustes en el mercado se producen por la rigidez salarial y el stock. Esto se da en mercados donde hay rigidez causada por las instituciones (por ejemplo, el salario mínimo), lo que hace que el salario no se ajuste a su nivel de equilibrio, ya que está por encima de dicho nivel y puede crear desempleo.

Por último, esta teoría habla del ambiente en el que se hace más productivo el trabajo. Es así como la productividad de los trabajadores, dado el capital y un entorno productivo adecuado, mejora el nivel de empleo y el salario; por esto se habla de que cada trabajador, según se pueda ajustar o no a su entorno, será más o menos productivo, por cuanto la productividad física de los factores permite mayores beneficios para la producción. La complementariedad entre tecnología y fuerza de trabajo es lo que determina los ingresos laborales. Por consiguiente, la productividad queda matizada no solo por la capacidad de trabajo, sino por un entorno productivo.

\section{Enfoque keynesiano}

Keynes considera que existen precios rígidos en el mercado; él habla de salarios rígidos que provocan que el mercado laboral no siempre se vacíe, lo que implica que haya desempleo involuntario, ya que los salarios no se ajustan rápidamente a los choques del mercado laboral. Además, la determinación de los salarios y el nivel de empleo son procesos diferentes, por ello, el equilibrio del que hablan los neoclásicos no es real, si los salarios no son capaces de igualar la oferta y la demanda de trabajo. En este sentido, para Keynes (1973), el desempleo viene determinado por la insuficiencia de la demanda efectiva, que vendría, a su vez, dada por un exceso de oferta en el mercado laboral y de bienes y servicios. El autor recurre a la intervención del Estado para que aumente la demanda agregada y así poder reducir el desempleo, ya que se incrementan la demanda de mano de obra y los salarios.

Keynes acepta que el salario real es igual al producto marginal del trabajo, como indica la teoría neoclásica, pero cuestiona la relación de la demanda laboral como función decreciente del salario, ya que el salario sería fijado por el volumen de empleo que viene dado por los niveles de inversión y de consumo, que son la 
demanda agregada. Es decir, la demanda agregada determina el nivel de empleo y este, el nivel de los salarios. Los cambios en la demanda agregada vienen dados por cambios en las expectativas de los empresarios sobre el futuro del mercado (Ruesga, Pérez, Ortiz, Lasierra, Trujillo \& Bichara, 2014).

\section{Teoría de los salarios de eficiencia}

Esta teoría se basa en el problema de que los salarios se mantienen en un nivel superior que el correspondiente con el pleno empleo, aun cuando haya crisis económica. La teoría de los salarios de eficiencia plantea que las empresas no se interesan por reducir los salarios reales, porque tienen una relación directa y positiva con la productividad de los trabajadores (Azariadis \& Stiglitz, 1983).

Los empresarios tienen una dificultad al no contar siempre con la información adecuada sobre la productividad real de los trabajadores (información asimétrica), así, las interacciones en el mercado laboral se convierten en un juego de señalización. El trabajador envía señales al empleador a través de su nivel de educación, la experiencia y el salario en el momento de solicitar el trabajo, y las firmas también envían una señal a los trabajadores más productivos, ofreciendo salarios más altos (Zuluaga \& Manzur, 2012). De no hacerlo, se genera un problema de selección adversa, en la medida en que solamente podrá atraer a los trabajadores menos productivos (Malcomson, 1981). Igualmente, ante una crisis, los empresarios no querrán reducir salarios, para no ahuyentar a los empleados más productivos.

Otro problema al que se enfrentan los empresarios hace énfasis en los costes de rotación laboral. Las firmas desean reducir la costosa rotación laboral, por ello prefieren ofrecer salarios más altos que los de equilibrio para retener a sus trabajadores (Stiglitz, 1984). Así mismo, en áreas con altas tasas de desempleo se pagarán salarios más bajos que en las áreas con altas tasas de empleo, para trabajadores con características similares (Blanchflower \& Oswald, 1995). En síntesis, esta teoría se basa en el comportamiento de los salarios reales y los problemas a los que los empresarios deben hacer frente en medio de las características de los trabajadores y el mercado.

Entre los planteamientos acerca de la manera como se determinan los salarios se observa el comportamiento del mercado y sus fallos, así como de los estímulos y expectativas de los trabajadores y empresarios. Lo anterior se recoge en las tres teorías descritas anteriormente, de allí se parte a un análisis más específico sobre cómo se caracteriza la oferta laboral, en este caso analizando el papel de la educación y la experiencia.

\section{EDUCACIÓN Y MERCADO LABORAL}

La relación existente entre educación y mercado de trabajo se concibe en el 
análisis de la oferta de mano de obra, y en ella se observa cómo se determinan las diferencias de la oferta laboral para diferentes o similares trabajos. La adquisición de habilidades para el mercado de trabajo ha sido un aspecto ampliamente estudiando y que sigue generando interés en los países, dada su gran importancia en pro del desarrollo económico y social.

En esta pesquisa se presentarán algunos postulados sobre la relación entre educación, experiencia y mercado laboral, partiendo de la teoría del capital humano como el eje principal de la relación entre educación-productividadsalarios. Después se muestran diferentes críticas a esta relación provenientes de otras teorías, para no solo observar la importancia de la educación, sino la problemática y el análisis de la experiencia, como fundamento en la capacitación y determinación salarial de las personas.

\section{Teoría del capital humano}

La teoría del capital humano estudia la conexión entre mayores cualificaciones, provenientes de la educación, y mayor productividad, que provocan mejores salarios. Por tanto, el nivel educativo y la experiencia que forjan esas cualificaciones supondrían un aspecto de vital importancia para cualquier economía. Lo anterior se basa en el planteamiento neoclásico sobre formación y remuneración salarial de los trabajadores.
La teoría ve la educación como una inversión y no como un gasto, en este caso es una inversión personal pensando, como en toda inversión, en mejores retribuciones futuras. Es por ello que las personas gastan en aspectos como salud y educación, dado que a mayores cualificaciones y mejor formación profesional, se esperan trabajos con mayores salarios para que sea rentable su inversión, con la que desean recuperar el dinero invertido en la formación y el costo de oportunidad por la formación adquirida.

Este concepto es relativamente nuevo, fue propuesto por Schultz (1961) y Becker (1964a). Schultz se centró en la educación como una inversión, ya que eleva la productividad individual del trabajo y con ello aumentan los niveles salariales en el futuro. Becker demostró empíricamente las correlaciones positivas que había entre cada nivel de educación con el salario, y concluyó que a mayor nivel educativo, mayor nivel salarial; además publicó que los países con un mayor nivel educativo eran los que se consideraban países desarrollados.

Schultz y Becker fundamentaron sus postulados en los aportes de Adam Smith y los desarrollos de la teoría del crecimiento económico de Solow (1956). De este modo, la combinación de mayores estudios, grados de educación y mayor experiencia llevan a mayores rendimientos representados en salarios más altos. Ante el planteamiento neoclásico de que el desempleo es voluntario y que hay 
homogeneidad en los trabajadores, pero que en realidad no se cumple, la teoría del capital humano considera que las diferencias salariales de los trabajadores vienen dadas por el nivel de cualificaciones de los individuos (heterogeneidad), además de que personas más cualificadas son más demandadas, así el desempleo ya no se considera voluntario.

La teoría del capital humano se ha relacionado con el crecimiento económico de forma reiterada desde Mankiw, Romer y Weil (1990), para quienes tiene una relación positiva que complementa el crecimiento de la economía, y es, por tanto, un factor esencial que falta en la teoría de crecimiento de Solow. Además, para Lucas (1988), el capital humano está relacionado con el crecimiento endógeno; sin embargo, es considerado como cualquier otro factor de la producción. Por último, para Azariadis y Drazen (1990), la educación es un factor relevante en el crecimiento económico, ya que es lo que permite acumular capital humano y este último se traduce en mayor crecimiento económico.

Por otro lado, no solo es importante invertir en la preparación de los individuos para mejorar su productividad, sino que hay que hacerlo de la mejor forma, es así como se debe invertir en mayor cobertura y calidad, pero también se deben orientar las inversiones hacia sectores con mayor productividad, que llevan consigo una mejora en el bienestar general de la sociedad (Mushkin, 1962). Uno de los aspectos que puede evidenciar el cambio de los retornos salariales en las últimas décadas es la escasez de mano de obra con altos niveles de formación en la segunda mitad del siglo XX, situación que ha cambiado con el mayor acceso a mayores niveles de educación de la población. Por tanto, los retornos de la educación han disminuido porque ya no hay tanta escasez de mano de obra cualificada y hay una desconexión entre la demanda de habilidades y la oferta de trabajadores cualificados.

Después de la formación por medio del sistema educativo se produce la de la trayectoria laboral, es así como para Becker (1964b) se diferencian dos tipos de formación de los individuos, uno general y otro específico. En el primero, aumenta la productividad de las personas de forma directa sin tener en cuenta las características del sector o empresa en la que trabaje o vaya a trabajar, es por esta razón que el coste de esta formación la asume el trabajador y no la empresa. La segunda forma se refiere a las capacidades que solo se pueden dar en un sector o empresa específica de la economía en que se halle la persona; por esta razón la empresa se encarga de los costes de esta formación. De igual manera, este autor propone una cofinanciación de la formación evitando costes altos de rotación laboral para las empresas, ya que perderían gran parte de su inversión en capacitación.

La teoría del capital humano tuvo gran éxito a mediados del siglo XX, pero a partir de la crisis del petróleo en 1979 se 
vieron desajustes entre la relación de la educación y la productividad en los países desarrollados, lo que permitió que desde diferentes corrientes de pensamiento se criticara la teoría del capital humano. A continuación, se describen algunas críticas y se muestra como se da esta relación entre educación y salarios para otros pensadores.

\section{Críticas a la teoría del capital humano}

\section{Teoría del credencialismo}

Esta teoría parte de que la productividad no viene dada por la educación en sí, sino por los títulos o credenciales que son los que permiten competir por un puesto de trabajo. Collins (1986) alude a ello como una crítica a la teoría del capital humano y niega la productividad del trabajo como beneficio de la educación, ya que el conocimiento para realizar la labor viene de la experiencia profesional más que de la educación.

Collins (1986) dice que las personas se educan solo porque la sociedad tiene imposiciones de costumbres (la forma de hablar, vestir, comportarse, etc.) que deben cumplir para desenvolverse en la misma, pero no como parte de un desarrollo verdadero de sus capacidades. En este sentido, el título (credencial) que los individuos reciben por su formación, solo les permite ascender de clase social, pero no demuestra fielmente que tenga las habilidades y conocimientos, ya que estos se dan con la experiencia.
Como se considera que la educación no aumenta la productividad, esta serviría como un instrumento para identificar individuos que ya son más productivos y capaces de antemano; por esta razón, la competencia no va por mayores salarios, sino guiada por mejores puestos de trabajo. Además, las personas más educadas suponen un menor coste de formación, por tanto, son más contratadas (Thurow, 1983).

Con base en lo anterior, la educación ayuda a seleccionar los mejores para ser formados, tal como plantea la teoría del filtro y la señalización (Spence, 1973; Arrow, 1973). Entonces, a mayor nivel educativo, será mejor la selección, debido a que las personas han pasado por varios obstáculos académicos y los han superado. La credencial que les supone esto, les permite ir por mejores salarios. Por eso, la educación cataloga a las personas, es decir, las filtra, y genera y da información al mercado de trabajo, es decir, señaliza.

Por último, Stiglitz (1975) dice que los fallos del mercado, como la información asimétrica, supone la intervención del Estado en la mayoría de mercados, pero en el mercado laboral el nivel educativo cumple esta función y permite dar solución al problema de información imperfecta. Por tanto, el nivel de educación de una persona debe suponer también mayores salarios, el problema es que la productividad permanece inalterable, ya que la educación no eleva 
la productividad, sino solo los salarios, por ello, acrecienta los problemas de desigualdad en la distribución de la renta nacional, en donde los menos educados se verán más afectados por tener menores salarios que quienes tienen más educación.

\section{Institucionalistas}

La teoría institucionalista también critica la teoría del capital humano en la relación entre educación, productividad y salario. Para esta teoría, lo que más importa no es la educación, sino la formación adquirida en el puesto de trabajo, es decir, la experiencia. Así mismo, rechaza los postulados de competencia perfecta, plantea que no hay muchas posibilidades de movilidad laboral y que hay información asimétrica en el mercado, que impide su buen funcionamiento afectando la seguridad de los trabajadores.

Igualmente, rechaza la idea de que las diferencias salariales se dan por diferentes cualificaciones, puesto que se dan por el poder de cada empresa de pagar salarios distintos a trabajadores con habilidades similares. Por ello, se plantea que cada empresa debe tener cierto control, por ejemplo, con gremios sindicales, que procuren mayor equidad salarial por competencias de trabajo (Kerr, 1985).

Así mismo, se piensa que las condiciones de las firmas en el mercado dan a los trabajadores cierta productividad, que permite eliminar sobrecostes que no influyen en la productividad; por tanto, si una empresa tiene poder de mercado, podrá reducir costes de producción (salarios). Por lo anterior, es necesario un mayor poder sindical para contrarrestar el poder de mercado de la empresa, que presione los salarios a la baja (Brown \& Medoff, 1989). La presión sindical logra aumentar los salarios y las empresas más grandes y competitivas consiguen mejor posicionamiento de mercado que les permite mejorar las condiciones de sus trabajadores. Por eso es muy importante manejar el contexto de mercado para mejorar los ingresos laborales.

También se encuentra la teoría del mercado dual del trabajo, generalizada por Piore (1983), para quien existen dos tramos en el mercado laboral. El sector primario, que tiene altas remuneraciones salariales, dando la posibilidad de ascenso en el trabajo y de generar mayor estabilidad en la trayectoria laboral, y el sector secundario, que cuenta con menores remuneraciones salariales, menores posibilidades de ascenso y es más inestable.

De igual forma, el sector primario se divide en superior e inferior; el primero formado por los profesionales y altos directivos que pueden tener mayor iniciativa, desarrollar más la creatividad y mantener estabilidad financiera por su salario, y el inferior que tiene menor movilidad dentro de la empresa y presenta salarios más bajos. El pertenecer a alguno de estos segmentos depende del nivel de cualificación de los trabajadores que, a su vez, depende de la situación social 
de los mismos (Piore, 1983). Por otro lado, el cambio tecnológico provoca la segmentación de los mercados, de este modo el dualismo se genera debido a que la productividad de los factores depende de la división del trabajo que, a su turno, depende de la dimensión del mercado y la demanda (Berger \& Piore, 1980).

Al reconocerse la diferenciación en grupos de los segmentos por nivel de cualificación, ya descritos, se permite el análisis de los denominados mercados internos de trabajo, en donde el fomento interno es el principal vehículo para tener mejor posición y salario. Doeringer y Piore (1985) definen el mercado interno de trabajo como aquel en que los precios y asignación de los puestos de trabajo se hallan bajo normas administrativas. Para estos mismos autores, esta idea contrasta con el llamado mercado externo de trabajo, en donde los precios y la asignación de los puestos de trabajo son controlados de forma directa por la dinámica económica.

La tecnología permite la aparición de estos mercados internos, por tanto, las empresas con mayor tecnología aumentan la demanda de mano de obra más cualificada. La empresa forma a estos trabajadores e incurre en un costo adicional que se traduce en una inversión para incrementar su productividad, por ello se evita la rotación externa (despidos) de estos trabajadores.

Dentro de esta teoría, la experiencia es un aspecto importante; en este caso la experiencia en las empresas posibilita el acceso al sector secundario, o también el acceso al sector superior del segmento primario para quienes cuentan con niveles educativos altos. En el primer caso se hará con bajos salarios y mayor inestabilidad, en el segundo con mejores salarios y estabilidad (Doeringger \& Piore, 1983).

En las nuevas teorías del mercado laboral se da la denominada de mercados transicionales. Se introduce el estudio del ciclo de vida de las personas y los problemas de inserción laboral (Ruesga et al., 2014). Dentro del ciclo de vida de los individuos se estudian eventos marcados en el tiempo, se observa la transición entre la educación y el trabajo, entre un empleo a otro (o distintos tipos de empleo), se contempla además la transición entre estar ocupado y desempleado, entre actividades con o sin remuneración monetaria y, por último, la transición desde el estar empleado al estar jubilado (Schmid, 2008). Estos niveles están asociados a actividades socialmente aceptadas y vistas como productivas, por lo cual se admiten distintos niveles de salario y permiten tener más posibilidades de acenso.

La educación ha sido el eje de las anteriores teorías, en el capital humano la educación ya no se ve como un gasto en consumo, sino como inversión, que por tanto tendrá retornos en el tiempo, reflejo de la mayor productividad y, por ende, mejores salarios. Para los críticos de esta teoría, como los credencialistas, la educación es una herramienta que da señales de cualificación, pero que no aumenta la 
productividad. Para los institucionalistas, la educación tiene mayor efecto en el sector primario que en el secundario, y es un factor importante para la transición del sector secundario al primario. En ellas, la experiencia juega un papel central de igual forma, pero más para los credencialistas e institucionalistas, que creen que la experiencia aporta más a la productividad que la educación en sí misma.

\section{ANÁLISIS EMPÍRICO}

El análisis que se hace está influenciado por otros estudios similares en cuanto a la problemática y a la metodología, pudiendo destacar a Buchinsky (1994) y a Katz y Kearney (2004) para Estados Unidos; Fitzenberger, Hujer, MaCurdy y Schnabel (2001) para Alemania; Nielsen y Rosholm (2001) para Zambia, y Freitas (2015) para Brasil.

Para Colombia se han hecho algunos estudios usando las regresiones cuantílicas, como en Vélez (2011) y López y Mora (2007), quienes explican la forma de uso de esta metodología; además están los trabajos de Flores y Peña (2010) y Galvis (2010), quienes usaron las regresiones cuantílicas para analizar las diferencias salariales por género en el país; también está el trabajo de Vargas (2011), quien usó este método para ver las desigualdades salariales por nivel educativo. Por último, en el estudio de Zárate (2003) se llevó a cabo, no solo el análisis de las desigualdades salariales, sino de los retornos de la educación y la experiencia con base en la teoría del capital humano por medio de las regresiones cuantílicas.

En general, se han estudiado las diferencias salariales, pero no tanto los retornos de la educación y experiencia y aun menos para ver la diferencia entre los jóvenes y adultos, que denota la problemática de baja inserción y bajo nivel empleo para jóvenes, además de bajos salarios para el nivel educativo para ambos grupos.

\section{Modelo}

Las ecuaciones se basan en el modelo de Mincer, que tradicionalmente se hace por mínimos cuadrados ordinarios, variaciones que explican Cahuc, Carcillo, Zylberberg y McCain (2014), sobre toda la muestra. En este caso se hicieron estimaciones por medio de la regresión cuantílica, pero cuyos modelos modifican la forma tradicional de hacer estas estimaciones. Las primeras tres ecuaciones muestran como tradicionalmente se estiman los retornos salariales, y las ecuaciones 4, 5, 6 y 7 son las que se estimarán en este artículo.

$$
\begin{gathered}
\text { linglabo }=\alpha_{0}+\alpha_{1}+\text { esc }+e \\
\text { linglabo }=\alpha_{0}+\alpha_{1}+\text { esc }+\alpha_{2} \text { expe }+\alpha_{3} \text { expe }^{2}+e \\
\text { linglabo }=\alpha_{0}+\alpha_{1}+\text { esc }+\alpha_{2} \text { esc }^{2}+\alpha_{3} \text { expe }+\alpha_{4} \text { expe }^{2}+e
\end{gathered}
$$


El salario se da como un logaritmo natural del ingreso laboral; esta transformación se ve justificada porque se aproxima a la distribución normal. En la ecuación 1, el logaritmo del salario (linglabo) se encuentra explicado por la educación (esc); en la ecuación 2, el salario viene explicado por la educación, la experiencia (expe) y la experiencia al cuadrado $\left(\right.$ expe $\left.^{2}\right)$, y en la ecuación 3 , el salario se explica por la educación, la educación al cuadrado $\left(e s c^{2}\right)$, experiencia y la experiencia al cuadrado.

El estimador $\alpha_{1}$ muestra el retorno de la educación sobre el ingreso (ecuaciones 1 y 2), el efecto de la experiencia en las ecuaciones 2 y 3 se ve como la derivada parcial entre el salario y la experiencia $\left(\alpha_{2}+2\left(\alpha_{3}\right.\right.$ expe $\left.) \%\right)$ para la ecuación 2 , y $\alpha_{3}+2\left(\alpha_{4}\right.$ expe $) \%$ para la ecuación 3.
Sobre la educación también se evalúan los efectos marginales decrecientes de la misma forma que la experiencia, esto en la ecuación 3, donde el efecto de la educación sobre el salario vendría determinado por la derivada parcial del salario entre la educación $\left(\left(\alpha_{1}+2\left(\alpha_{2}\right.\right.\right.$ esc $\left.\left.) \%\right)\right)$.

La presencia de rendimientos decrecientes de la educación no se ha abordado constantemente, pero es un tema trabajado desde el inicio de la teoría del capital humano, como muestra Selowsky (1968), quien aplica este principio de rendimientos decrecientes de la educación (niveles de educación: primaria, secundaria y universitaria) sobre el salario.

Las ecuaciones por regresión cuantílica son las siguientes:

linglabo $=\alpha_{0}+\alpha_{1}+$ esc $+\alpha_{2}$ sexo $+\alpha_{3}$ contrato $+\alpha_{4}$ dptotrabaja $+\alpha_{5}$ rama2d $+e$ [4]

linglabo $=\alpha_{0}+\alpha_{1}$ expe $+\alpha_{2}$ expe $e^{2}+\alpha_{3}$ sexo $+\alpha_{4}$ contrato $+\alpha_{5}$ dptotrabaja $+\alpha_{6}$ rama2d $+e$

linglabo $=\alpha_{0}+\alpha_{1}$ expe $+\alpha_{2}$ esc $+\alpha_{3}$ esc $^{2}+\alpha_{4}$ expe $^{2}+\alpha_{5}$ sexo $+\alpha_{6}$ contrato

$+\alpha_{7}$ dptotrabaja $+\alpha_{8}$ rama2d $+e$

linglabo $=\alpha_{0}+\alpha_{1}$ escexpe $+\alpha_{2}$ escexpe ${ }^{2}+\alpha_{3}$ sexo $+\alpha_{4}$ contrato

$+\alpha_{5}$ dptotrabaja $+\alpha_{6}$ rama2d $+e$ 
En estas ecuaciones se determina el salario, no solo por educación y experiencia, sino además se observan las variables sexo (genero), contrato (tener contrato), dpto (departamento donde trabaja) y rama2d (rama de actividad económica). Los $\alpha_{i}$ significan que por cada aumento de las variables explicativas, el ingreso variará un $\alpha_{i} \%$. Es decir, por cambios en las variables de nivel se da un cambio en el ingreso que está en logaritmo, de allí que la variación sea porcentual. Los efectos marginales decrecientes se analizan de forma similar a los demás modelos; se ven como la derivada parcial entre el ingreso y la experiencia $\left(\alpha_{i}+2\left(\alpha_{i}\right.\right.$ expe $\left.) \%\right)$ y $\alpha_{i}+$ $2\left(\alpha_{i}\right.$ esc $) \%$. Por tanto, una variación en la experiencia y educación significará un cambio en $\left(\alpha_{i}+2\left(\alpha_{i}\right.\right.$ expe $\left.) \%\right)$ y $\alpha_{i}+2\left(\alpha_{i}\right.$ $\left(\alpha_{i}+2\left(\alpha_{i}\right.\right.$ expe $) \%$.

En la ecuación 7 se ve el efecto conjunto de la educación y la experiencia; en ella el verdadero efecto se vería de nuevo en la derivada parcial del salario sobre escexpe. $\left(\alpha_{1}+2\left(\alpha_{2}\right.\right.$ escexpe $) \%$.

\section{Datos}

Las variables se toman de la Gran Encuesta Integrada de Hogares ${ }^{1}$ del $\mathrm{DANE}^{2}$ para el año 2015. El salario viene determinado por variable ingreso laboral, debido a que en la encuesta el ingreso laboral está definido por los ingresos en especie o por los subsidios, o por ambos, y el salario solo presenta el ingreso monetario devengado por el trabajo. La educación viene dada como años de escolaridad (esc), siendo esta expresada como el número de años de estudio que posee cada individuo (Barro \& Lee, 2001). En cuanto a la experiencia, esta se tomará como la suma de la duración en años del empleo anterior y actual. La variable género muestra el efecto de ser hombre o mujer en el salario y viene determinada como una dummy (hombre $=1 \mathrm{y}$ mujer $=0$ ), la variable contrato mide qué tanto incide el tener un contrato en el salario, también viene determinada como una dummy (tener contrato $=1$ y no tener contrato $=0$ ).

Así mismo, las variables de departamento donde trabaja (dptotrabaja) y rama de actividad económica (rama2d) miden el efecto de estar en una zona geográfica o en una actividad específica para tener mayor o menor salario. El aspecto regional no se trabaja a fondo en este estudio, solo se hace de forma generalizada, para referenciar las zonas de mayor impacto en el salario para jóvenes y adultos. La zona donde se trabaja, sin embargo, es de vital importancia, dada la relación que se da entre productividad y zona geográfica. Como explica Knight (1979), se da una relación más fuerte entre productividad y educación en las grandes ciudades, en la que hay mayor competencia por salarios y puestos de trabajo. En síntesis, las

\footnotetext{
http://www.dane.gov.co/

Departamento Administrativo Nacional de Estadística.
} 
regiones más productivas atraerán mano de obra más cualificada con mejores salarios. Por ello se toma no la región donde nació el trabajador, sino la región donde efectivamente trabaja.

\section{Metodología}

La formación de capital humano se relaciona con dos variables, educación y experiencia. Como se vio antes, el mayor énfasis se da en que la tasa de retorno se halla en la educación; es decir, se espera que un año de educación provoque un aumento en la tasa de ganancia (salario). La experiencia también es parte de ello, pero no se ha analizado tanto como la educación.

El segundo grupo de modelos se aborda mediante la regresión cuantílica. Esta fue generalizada por Koenker y Bassett (1978). En el modelo tradicional (MCO), los errores se toman como una sucesión $\left(\varepsilon_{n}\right)$ de variables aleatorias que son independientes e idénticamente distribuidas con media igual a cero. Sin embargo, no siempre se cumple el supuesto de normalidad, ya que hay distribuciones asimétricas; por ello Koenker y Bassett introducen las regresiones cuantílicas, para dar solución a este problema de asimetría. Con su trabajo demostraron que los estimadores por regresiones cuantílicas son más eficientes.

Otra diferencia sustancial radica en que en el modelo clásico se pretende minimizar la suma de los errores al cuadrado, y se basa en la media para los cálculos; en la regresión cuantílica se minimiza la suma de los errores absolutos ponderados para el efecto de las asimetrías, y se usan los cuantiles como estimadores. La regresión cuantílica descrita a continuación se basa en los trabajos de Koenker y Bassett (1978) y Buchinsky (1998) en cuanto a la metodología, y Buchinsky (1994) y Zárate (2003), por el énfasis en sus trabajos en los determinantes diferencias salariales. El modelo cuantílico puede partir de una distribución con $x$ elementos $x, x^{2}, \ldots, x^{\mathrm{n}} \in \mathbf{R}^{\mathrm{x}} \mathrm{y} n$ valores reales $z_{1}, z_{2}, \ldots$, $z_{n}$ En donde el problema de optimización queda definido como:

$$
\min f(\partial)=\sum_{i=1}^{n}\left(y_{i}-\partial^{T} x^{i}\right)^{2}
$$

Así mismo, de la ecuación 8 se puede obtener:

$$
\begin{gathered}
\hat{\partial}_{t}=\underset{\varepsilon_{T} \in \mathbf{R}}{\arg \min }\left\{\sum_{y_{i} \geq \varepsilon_{T}} T\left|y_{i}-\partial_{t}^{T} x_{i}\right|+\sum_{y_{i}<\varepsilon_{T}}(1-T)\left|y_{i}-\partial_{t}^{T} x_{i}\right|\right\} \\
\hat{\partial}_{t}=\underset{\varepsilon_{T}}{\arg \min } \sum_{i=1}^{n} \rho_{T}\left(y_{i}-\partial_{t}^{T} x_{i}\right) \\
\hat{\partial}_{t}=\underset{\varepsilon_{T} \in \mathbf{R}}{\arg \min } \sum_{i=1}^{n} \rho_{T}\left(\varepsilon_{T}\right)
\end{gathered}
$$


Donde $T$ es un valor entre 0 y $1,(T \in$ $(0,1))$ y $\rho_{\mathrm{T}}$ es la función de chequeo que puede expresarse como:

$$
\rho_{T}\left(\varepsilon_{i}\right)=\left\{\begin{aligned}
T \varepsilon_{i}, & \text { if } \varepsilon_{i}>0 \\
(1-T) \varepsilon_{i}, & \text { if } \varepsilon_{i} \leq 0
\end{aligned}\right.
$$

En la ecuación 9 se puede ver que todas las observaciones mayores a $\partial^{T}{ }_{t} X_{i}$ son estimadas por $\partial_{t} x$ con peso en $T$, y en las menores $x \partial_{t}$ con peso en $(1-T)$. También se ven las observaciones mayores que el valor absoluto de la diferencia entre las observaciones y el valor óptimo, las cuales tienen una ponderación de orden $T$ y las observaciones menores tienen una ponderación de orden $(1-T)$.

Como se dijo anteriormente, el análisis se hizo mediante regresiones cuantílicas para evaluar los retornos de la educación y experiencia, entre otras variables, por cuantiles. Se testearon 4 modelos, que se dividieron en jóvenes y adultos para los cuantiles 10, 25, 50, 75, y 90 para cada grupo. Las estimaciones se hicieron con el programa STATA (Data Analysis and Statistical Sofware); el grupo de jóvenes se conformó por personas entre los 18 y 28 años de edad, que normalmente se considera como etapa de juventud en la trayectoria laboral, y los adultos entre los 29 y 62 años de edad, que es el periodo entre la adultez y la jubilación.

En las ecuaciones mincerianas, sea por MCO o por regresiones cuantílicas, suele hablarse del problema de sesgos de especificación por omisión de variables relevantes, refiriéndose especialmente a las capacidades innatas de las personas, que daría una ventaja sobre otras personas en cuanto a habilidades y que repercutiría en mejores salarios. Esta variable no es observable por parte de los demandantes de trabajo. En este sentido se han realizado varios estudios que muestran que las habilidades innatas sí tienen impacto sobre la educación y los retornos salariales.

Algunos autores sostienen que las diferencias de rentas observadas entre individuos son únicamente resultado de un nivel de capacidad innata individual, es decir, la educación solo genera la información al empresario sobre las habilidades de estos, pero las personas más inteligentes y disciplinadas tienden a tener mayores niveles de educación. Así, los individuos más hábiles obtendrían mejores resultados en el ámbito laboral. En este caso, las tasas de rendimiento obtenido con la estimación de una ecuación de salarios sobrestimarían el rendimiento de la inversión educativa, ya que una proporción sería atribuible a la capacidad individual.

Cabe aclarar que la dificultad del contraste empírico de estas capacidades innatas impide ver con claridad el verdadero efecto de esta variable en los salarios. Se han estudiado varias formas de cuantificar estas capacidades, la más usada es la de métodos de análisis psicológico, como en Taubman y Wales (1972) y Blackburn y Neumark (1995), donde se encontró que la capacidad innata sí afecta los salarios. Otros estudios donde 
se aplicaron algunas baterías de pruebas psicológicas, Boissiere, Knight y Sabot (1985), encontraron que las capacidades innatas no afectan los retornos salariales y, por tanto, no habría sesgo por omisión de variable.

Los resultados muestran que en el modelo uno el ser hombre no tiene significancia estadística a la hora de tener mayores ingresos, en la mayoría de casos el coeficiente fue negativo en los jóvenes, es decir, las mujeres tenían mayor propensión a un mayor salario por el hecho de ser mujeres; $y$ en el grupo de los adultos el efecto fue positivo, es decir, los hombres tienen una propensión a mayores salarios que las mujeres, pero en ningún caso fue estadísticamente significativa.
La variable contrato se comporta de forma similar para ambos grupos. Primero, en los dos cuartiles inferiores ( 10 y 25$)$ tiene un efecto muy grande sobre el ingreso, y cae notoriamente desde el cuartil 50; vale anotar que, en todos los casos, menos en el cuartil 90, el efecto fue negativo, además en el cuartil 90 en ambos grupos no es estadísticamente significativa. La variable educación es estadísticamente significativa en los cuartiles 19 y 90 para jóvenes y en todos los cuartiles para adultos, esto es, la educación no es significativa a la hora de aspirar a los salarios más altos de la distribución de los jóvenes. Esto significa que los retornos de la educación comienzan a verse después de que se deja de considerar joven en el mercado laboral.

Tabla 1. Retornos de la educación para los jóvenes por cuantiles. 2015. Ecuación 4

\begin{tabular}{|l|r|r|r|r|r|}
\hline & \multicolumn{1}{|c|}{$\mathbf{q ( 1 0 )}$} & $\mathbf{q}(\mathbf{2 5})$ & $\mathbf{q ( 5 0 )}$ & $\mathbf{q}(\mathbf{7 5})$ & $\mathbf{q ( 9 0 )}$ \\
\hline \multirow{2}{*}{ Constante } & 13,67591 & 14,66035 & 13,76056 & 14,73099 & 16,64773 \\
\cline { 2 - 6 } & 0.000 & 0.000 & 0.000 & 0.000 & 0.000 \\
\hline \multirow{2}{*}{ Género } & $-0,0097385$ & 0,0254894 & 0,0057052 & $-0,0143802$ & $-0,013955$ \\
\cline { 2 - 6 } & 0,671 & 0.168 & 0.346 & 0.265 & 0.532 \\
\hline \multirow{2}{*}{ Contrato } & $-0,9380232$ & -1.011 .107 & $-0,1921208$ & $-0,0858816$ & 0,0384018 \\
\cline { 2 - 6 } & 0.000 & 0.000 & 0.000 & 0.000 & 0.097 \\
\cline { 2 - 6 } & 0,0100054 & 0,0055735 & 0,0018037 & 0,003101 & 0,0086038 \\
\hline
\end{tabular}

Fuente: elaboración propia a partir de las regresiones cuantílicas. (Ver anexo). 
Tabla 2. Retornos de la educación para los adultos por cuantiles. 2015

Ecuación 4

\begin{tabular}{|l|r|r|r|r|r|}
\hline & $\mathbf{q ( 1 0 )}$ & $\mathbf{q}(\mathbf{2 5})$ & $\mathbf{q ( 5 0 )}$ & $\mathbf{q}(\mathbf{7 5 )}$ & $\mathbf{q ( 9 0 )}$ \\
\hline \multirow{2}{*}{ constante } & 14,49557 & 14,62497 & 13,88955 & 15,11928 & 17,31808 \\
\cline { 2 - 6 } & 0.000 & 0.000 & 0.000 & 0.000 & 0.000 \\
\hline \multirow{2}{*}{ Género } & 0,0104938 & 0,0113778 & 0,0025186 & $-0,0015695$ & 0,0090621 \\
\cline { 2 - 6 } & 0.693 & 0.336 & 0.508 & 0.863 & 0.602 \\
\hline \multirow{2}{*}{ Contrato } & $-1,57591$ & $-0,9533762$ & $-0,1824032$ & $-0,1170885$ & $-0,0029681$ \\
\hline \multirow{2}{*}{ Esc } & 0.000 & 0.000 & 0.000 & 0.000 & 0.870 \\
\cline { 2 - 6 } & 0,0065632 & 0,0043826 & 0,0015768 & 0,0031439 & 0,0048059 \\
\hline
\end{tabular}

Fuente: elaboración propia a partir de las regresiones cuantílicas. (Ver anexo). En el segundo modelo (ecuación 5) se observa que de nuevo la variable género no es estadísticamente significativa en ningún grupo, y su efecto sobre el ingreso es positivo en los cuartiles medio-bajos del 10 al 50; es decir, que las mujeres, se presume, pueden tener una propensión a tener mayores salarios que los hombres en la parte más alta de la distribución. Además, la variable contrato de nuevo presenta efectos muy altos en los cuartiles inferiores ( 25 y 50 ) y va perdiendo relevancia a medida que aumentan los rangos, tanto para jóvenes como para adultos. La variable experiencia es significativa en todos los momentos, el mayor aumento proporcional del ingreso por cada año adicional de experiencia se da en los cuantiles 10, 25 y 90, y el efecto disminuye notoriamente en los cuartiles 50 y 75 para los dos grupos.

En el segundo modelo (ecuación 5) se observa que de nuevo la variable género no es estadísticamente significativa en ningún grupo, y su efecto sobre el ingreso es positivo en los cuartiles medio-bajos del 10 al 50; es decir, que las mujeres, se presume, pueden tener una propensión a tener mayores salarios que los hombres en la parte más alta de la distribución. Además, la variable contrato de nuevo presenta efectos muy altos en los cuartiles inferiores $(25 \mathrm{y}$ 50 ) y va perdiendo relevancia a medida que aumentan los rangos, tanto para jóvenes como para adultos. La variable experiencia es significativa en todos los momentos, el mayor aumento proporcional del ingreso por cada año adicional de experiencia se da en los cuantiles 10, 25 y 90, y el efecto disminuye notoriamente en los cuartiles 50 y 75 para los dos grupos. 
Retornos salariales para Colombia, un análisis cuantílico

Cristian Darío Castillo Robayo - Julimar Da Silva Bichara - Manuel Pérez Trujillo

Tabla 3. Retornos de la experiencia para los jóvenes por cuantiles. 2015 Ecuación 5

\begin{tabular}{|l|r|r|r|r|r|}
\hline \multirow{2}{*}{ Constante } & $\mathbf{q ( 1 0 )}$ & $\mathbf{q ( 2 5 )}$ & $\mathbf{q ( 5 0 )}$ & $\mathbf{q ( 7 5 )}$ & $\mathbf{q ( 9 0 )}$ \\
& 13,51904 & 14,58829 & 13,70286 & 14,60084 & 16,4501 \\
\cline { 2 - 6 } & 0.000 & 0.000 & 0.000 & 0.000 & 0.000 \\
\hline \multirow{2}{*}{ Género } & 0,0042847 & 0,0315838 & 0,008197 & $-0,0095722$ & $-0,0063221$ \\
\cline { 2 - 6 } & 0.848 & 0.089 & 0.163 & 0,444 & 0.771 \\
\hline \multirow{2}{*}{ Expe } & $-1,109309$ & $-1,291364$ & -0.2429864 & -0.2051981 & -.01307613 \\
\cline { 2 - 6 } & 0.000 & 0.000 & 0.000 & 0.000 & 0.000 \\
\hline \multirow{2}{*}{ Expe2 } & 0,0053482 & 0,0053828 & 0,0017228 & 0,0038155 & 0,0044002 \\
\cline { 2 - 6 } & 0.000 & 0.000 & 0.000 & 0.000 & 0.000 \\
\hline \multirow{2}{*}{ Efecto real expe } & 0,0034014 & 0,0033403 & 0,0010762 & .0024367 & 0,0030583 \\
& 0.000 & 0.000 & 0.000 & 0.000 & 0.000 \\
\hline
\end{tabular}

Fuente: elaboración propia a partir de las regresiones cuantílicas. (Ver anexo).

Tabla 4. Retornos de la experiencia para los adultos por cuantiles. 2015 Ecuación 5

\begin{tabular}{|l|r|r|r|r|r|}
\hline & \multicolumn{1}{|c|}{$\mathbf{q ( 1 0 )}$} & \multicolumn{1}{|c|}{$\mathbf{q ( 2 5 )}$} & $\mathbf{q ( 5 0 )}$ & $\mathbf{q ( 7 5 )}$ & $\mathbf{q ( 9 0 )}$ \\
\hline \multirow{2}{*}{ Constante } & 14,15620 & 14,36424 & 13,80009 & 14,90163 & 17,01155 \\
\cline { 2 - 6 } & 0.000 & 0.000 & 0.000 & 0.000 & 0.000 \\
\hline \multirow{2}{*}{ Género } & 0,0092559 & 0,0127759 & 0,0030206 & $-0,0020546$ & 0,0079883 \\
\cline { 2 - 6 } & $-1,862816$ & $-1,089467$ & -0.2229489 & -0.2256955 & $-0,1990148$ \\
\cline { 2 - 6 } & 0.000 & 0.000 & 0.000 & 0.000 & 0.000 \\
\hline \multirow{2}{*}{ Expe } & 0,0089718 & 0,048981 & 0,0015482 & 0,0035873 & 0,0052094 \\
\cline { 2 - 6 } & 0.000 & 0.000 & 0.000 & 0.000 & 0.000 \\
\cline { 2 - 6 } & $-0,0000143$ & $-8.25 \mathrm{e}-06$ & $-2.59 \mathrm{e}-06$ & $-5.49 \mathrm{e}-06$ & $-6.60 \mathrm{e}-06$ \\
\hline \multirow{2}{*}{ Efecto real expe } & 0,0056064 & 0,0029638 & 0,0009409 & 0,0022993 & 0,0036613 \\
& 0.000 & 0.000 & 0.000 & 0.000 & 0.000 \\
\hline
\end{tabular}

Fuente: elaboración propia a partir de las regresiones cuantílicas. (Ver anexo). 
En el tercer modelo (ecuación 6) se evalúa el efecto de la educación y la experiencia, y sus rendimientos marginalmente decrecientes, en la misma regresión. La variable género no es significativa en ningún momento y de nuevo presenta positivos en los cuartiles 25 y 50 de los jóvenes y en todos los cuartiles de los adultos. La variable contrato muestra efectos muy altos en los cuartiles 10 y 25 para los jóvenes, pero siempre negativo, y a partir de allí cae notoriamente y va disminuyendo su valor progresivamente; para los adultos, el efecto es alto en todos los cuartiles menos en el 90, y siempre negativo. Para los jóvenes, la variable educación es significativa en el primer cuartil, y la experiencia en todos los cuantiles. Para los adultos, la educación es estadísticamente significativa en todos los cuartiles al igual que la experiencia.

Además, la educación representa mayores retornos para los jóvenes en todos los cuartiles menos en el 50; pero vale la pena recordar que solo es estadísticamente significativo en el primer cuartil; en cuanto a la experiencia, representa mayores retornos en los cuartiles medios (25,50 y 75$)$ para los jóvenes, y para los adultos en los extremos (10 y 90), así mismo dentro de cada grupo los efectos mayores se ven en el cuartil 10 y 90 .

La educación muestra rendimientos más altos que la experiencia tanto para jóvenes como para adultos en todos los cuantiles, es decir, tener un año más de educación hace que aumente más el salario que tener un año más de experiencia. Sin embargo, para los jóvenes, al no haber significación estadística, sino solo en el primer cuartil, la experiencia toma la relevancia central en explicar los retornos salariales.

En el último modelo (ecuación 7) se evalúa el efecto conjunto de tener más educación y experiencia. La variable género no es significativa en ningún momento ni para jóvenes ni para adultos. La variable contrato presenta efectos altos en los cuartiles inferiores ( 25 y 50 ) y va perdiendo efecto a medida que aumentan los rangos, tanto para jóvenes como para adultos, pero manteniendo el signo negativo. El efecto conjunto de experiencia y educación (escexpe) es significativo para jóvenes y adultos en todos los cuantiles, el efecto es mayor en los cuantiles 10 y 90 para jóvenes y adultos y menor en el 50. El efecto en los jóvenes es superior al de los adultos en todos los cuartiles menos en el 10 y 90 . 
Retornos salariales para Colombia, un análisis cuantílico

Cristian Darío Castillo Robayo - Julimar Da Silva Bichara - Manuel Pérez Trujillo

Tabla 5. Retornos de la educación y experiencia para los jóvenes por cuantiles. 2014 Ecuación 6

\begin{tabular}{|c|c|c|c|c|c|}
\hline & $q(10)$ & $q(25)$ & $q(50)$ & $q(75)$ & $q(90)$ \\
\hline \multirow[t]{2}{*}{ Constante } & 13,37751 & 14,38982 & 13,67824 & 14,57334 & 16,46446 \\
\hline & 0.000 & 0.000 & 0.000 & 0.000 & 0.000 \\
\hline \multirow[t]{2}{*}{ Género } & $-0,0033312$ & 0,0303604 & 0,0063534 & $-0,0129721$ & $-0,0119693$ \\
\hline & 0.883 & 0.094 & 0.286 & 0.305 & 0.588 \\
\hline \multirow[t]{2}{*}{ Contrato } & $-1,111358$ & $-1,171696$ & $-0,2439066$ & $-0,2045408$ & $-0,1302848$ \\
\hline & 0.000 & 0.000 & 0.000 & 0.000 & 0.000 \\
\hline \multirow[t]{2}{*}{ Esc } & 0,0194617 & 0,0112616 & 0,0022399 & $-0,0014776$ & 0,0028088 \\
\hline & 0.279 & 0.437 & 0.631 & 0.881 & 0.870 \\
\hline \multirow[t]{2}{*}{ Esc2 } & $-0,0004527$ & $-0,0002686$ & $-0,0000221$ & 0,0002111 & 0,0002661 \\
\hline & 0.581 & 0.688 & 0.918 & 0.643 & 0.740 \\
\hline \multirow[t]{2}{*}{ expe } & 0,0053398 & 0,0051065 & 0,0017242 & 0,0038038 & 0,0044183 \\
\hline & 0.000 & 0.000 & 0.000 & 0.000 & 0.000 \\
\hline \multirow[t]{2}{*}{ Expe2 } & $-8.28 \mathrm{e}-06$ & $-8.03 e-06$ & $-2.75 e-06$ & $-5.85 e-06$ & $-5.71 e-06$ \\
\hline & 0.000 & 0.000 & 0.000 & 0.000 & 0.000 \\
\hline \multirow{2}{*}{$\begin{array}{l}\text { Efectoreal educ (derivada } \\
\text { parcial del salario entre la } \\
\text { educación) }\end{array}$} & 0,0100584 & 0,0056816 & 0,0017815 & 0,0029075 & 0,0083359 \\
\hline & 0.011 & 0.068 & 0.079 & 0.176 & 0.025 \\
\hline \multirow{2}{*}{$\begin{array}{l}\text { Efecto real expe } \\
\text { (derivada parcial del } \\
\text { salario entre } \\
\text { la experiencia) }\end{array}$} & 0,0033983 & 0,0032224 & 0,0010782 & 0,0024313 & 0,0030794 \\
\hline & 0.000 & 0.000 & 0.000 & 0.000 & 0.000 \\
\hline
\end{tabular}

Fuente: elaboración propia a partir de las regresiones cuantílicas. (Ver anexo). 
Tabla 6. Retornos de la educación y experiencia para los adultos por cuantiles. 2015 Ecuación 6

\begin{tabular}{|c|c|c|c|c|c|}
\hline & $q(10)$ & $q(25)$ & $q(50)$ & $q(75)$ & $q(90)$ \\
\hline \multirow[t]{2}{*}{ Constante } & 14,014360 & 14,3358 & 13,79629 & 14,89286 & 16,97198 \\
\hline & 0.000 & 0.000 & 0.000 & 0.000 & 0.000 \\
\hline \multirow[t]{2}{*}{ Género } & 0,007286 & 0,0122006 & 0,0027924 & $-0,0016266$ & 0,0080055 \\
\hline & 0.782 & 0.294 & 0.456 & 0.855 & 0.640 \\
\hline \multirow[t]{2}{*}{ Contrato } & $-1,8500850$ & $-1,0870020$ & $-2,2404360$ & $-2,2658930$ & $-0,1978658$ \\
\hline & 0.000 & 0.000 & 0.000 & 0.000 & 0.000 \\
\hline \multirow[t]{2}{*}{ Esc } & 0,0085056 & 0,0100479 & 0,0033412 & 0,0082055 & 0,0148896 \\
\hline & 0.457 & 0.047 & 0.041 & 0.035 & 0.042 \\
\hline \multirow[t]{2}{*}{ Esc2 } & $-0,0000693$ & $-0,0002728$ & $-0,0000853$ & $-0,0002465$ & $-0,000497$ \\
\hline & 0.898 & 0.256 & 0.276 & 0.188 & 0.159 \\
\hline \multirow[t]{2}{*}{ Expe } & 0,0089389 & 0,0048781 & 0,0015567 & 0,0036124 & 0,0051829 \\
\hline & 0.000 & 0.000 & 0.000 & 0.000 & 0.000 \\
\hline \multirow[t]{2}{*}{ Expe2 } & $-0,0000143$ & $-8.19 e-06$ & $-2.60 \mathrm{e}-06$ & $-5.53 e-06$ & $-6.57 e-06$ \\
\hline & 0.000 & 0.000 & 0.000 & 0.000 & 0.000 \\
\hline \multirow{2}{*}{$\begin{array}{l}\text { Efectoreal educ (derivada } \\
\text { parcial del salario entre la } \\
\text { educación) }\end{array}$} & 0,0070659 & 0,0043823 & 0,0015693 & 0,0030854 & 0,0045672 \\
\hline & 0.015 & 0.001 & 0.000 & 0.002 & 0.015 \\
\hline \multirow{2}{*}{$\begin{array}{l}\text { Efecto real expe(derivada } \\
\text { parcial del salario entre la } \\
\text { experiencia) }\end{array}$} & 0,0055891 & 0,002956 & 0,0009463 & 0,002314 & 0,003642 \\
\hline & 0.000 & 0.000 & 0.000 & 0.000 & 0.000 \\
\hline
\end{tabular}

Fuente: elaboración propia a partir de las regresiones cuantílicas. (Ver anexo).

Tabla 7. Retornos del efecto combinado entre la educación y experiencia para los jóvenes por cuantiles. 2015. Ecuación 7

\begin{tabular}{|c|c|c|c|c|c|}
\hline & $q(10)$ & $q(25)$ & $q(50)$ & $q(75)$ & $q(90)$ \\
\hline \multirow[t]{2}{*}{ Constante } & 13,57550 & 14,52424 & 13,71602 & 14,61857 & 16,55382 \\
\hline & 0.000 & 0.000 & 0.000 & 0.000 & 0.000 \\
\hline \multirow[t]{2}{*}{ Género } & -.008101 & .0241382 & .0042703 & -.0184064 & -.0179338 \\
\hline & 0.720 & 0.182 & 0.472 & 0.144 & 0.415 \\
\hline \multirow[t]{2}{*}{ Contrato } & $-1,104607$ & -1.161 .718 & -.2407454 & -.1954043 & -.1209345 \\
\hline & 0.000 & 0.000 & 0.000 & 0.000 & 0.000 \\
\hline \multirow[t]{2}{*}{ Escexpe } & .0004027 & .0003786 & .0001268 & .0002818 & .0003508 \\
\hline & 0.000 & 0.000 & 0.000 & 0.000 & 0.000 \\
\hline \multirow[t]{2}{*}{ Escexpe2 } & $-4.73 e-08$ & $-4.54 \mathrm{e}-08$ & $-1.54 \mathrm{e}-08$ & $-3.32 e-08$ & $-3.55 e-08$ \\
\hline & 0.000 & 0.000 & 0.079 & 0.157 & 0.023 \\
\hline \multirow[t]{2}{*}{ Efecto real escexpe } & .0002885 & .0002689 & .0000897 & .0002016 & .000265 \\
\hline & 0.000 & 0.000 & 0.000 & 0.000 & 0.000 \\
\hline
\end{tabular}

Fuente: elaboración propia a partir de las regresiones cuantílicas. (Ver anexo). 
Tabla 8. Retornos del efecto combinado entre la educación y experiencia para los adultos por cuantiles. 2015. Ecuación 7

\begin{tabular}{|c|c|c|c|c|c|}
\hline & $q(10)$ & $q(25)$ & $q(50)$ & $q(75)$ & $q(90)$ \\
\hline \multirow[t]{2}{*}{ Constante } & 14,25329 & 14,49175 & 13,84765 & 15,00285 & 17,12274 \\
\hline & 0.000 & 0.000 & 0.000 & 0.000 & 0.000 \\
\hline \multirow[t]{2}{*}{ Género } & .0060529 & .0116302 & .0026154 & -.0019103 & .0078908 \\
\hline & 0.819 & 0.319 & 0.487 & 0.831 & 0.646 \\
\hline \multirow[t]{2}{*}{ Contrato } & $-1,795040$ & $-1,061721$ & -.2160082 & -.2032891 & -.1566908 \\
\hline & 0.000 & 0.000 & 0.000 & 0.000 & 0.000 \\
\hline \multirow[t]{2}{*}{ Escexpe } & .0005671 & .0003091 & .0000986 & .000241 & .0003757 \\
\hline & 0.000 & 0.000 & 0.000 & 0.000 & 0.000 \\
\hline \multirow[t]{2}{*}{ Escexpe2 } & $-6.32 e-08$ & $-3.63 e-08$ & $-1.15 e-08$ & $-2.64 \mathrm{e}-08$ & $-3.52 e-08$ \\
\hline & 0.000 & 0.000 & 0.079 & 0.157 & 0.023 \\
\hline \multirow[t]{2}{*}{ Efecto real escexpe } & .0004145 & .0002214 & .0000709 & .0001772 & .0002906 \\
\hline & 0.000 & 0.000 & 0.000 & 0.000 & 0.000 \\
\hline
\end{tabular}

Fuente: elaboración propia a partir de las regresiones cuantílicas. (Ver anexo).

Además de los análisis anteriores se realizó una regresión intercuantílica para ver las diferencias entre cuantiles de cada grupo. Las diferencias planteadas son entre: (90-Q10) (Q90-Q75), (Q90-Q50), (Q75-Q25), (Q50-Q25) Y (Q25-Q10). En las tablas 9 y 10 se observan los resultados del análisis de la educación para jóvenes y adultos. Se dio el mismo efecto para jóvenes y adultos, pues no se nota que la educación genere la diferencia salarial entre los salarios más altos y más bajos (90-10) y (75-25). Las diferencias se hallan en la parte alta y media (90-75) baja (25-10) y (media $50-25$.)

Tabla 9. Diferencias intercuantílicas educación jóvenes

\begin{tabular}{|l|r|r|r|r|r|r|}
\hline & \multicolumn{1}{|c|}{$\mathbf{2 5 - 1}$} & \multicolumn{1}{|c|}{$\mathbf{5 - 2 5}$} & \multicolumn{1}{|c|}{$\mathbf{9 - 1}$} & \multicolumn{1}{|c|}{$\mathbf{7 5 - 2 5}$} & \multicolumn{1}{|c|}{$\mathbf{9 - 7 5}$} & $\mathbf{9 - 5}$ \\
\hline \multirow{2}{*}{ esc } & $-0,008593$ & $-0,016105$ & $-0,0040236$ & $-0,0070239$ & 0,0115932 & 0,0206744 \\
\cline { 2 - 7 } & 0,093 & 0.001 & 0.419 & 0.163 & 0.000 & 0.000 \\
\hline \multirow{2}{*}{ constante } & 0,9184341 & 0,6597534 & 2,366962 & 0,983018 & 0,4655104 & 0,788775 \\
\cline { 2 - 7 } & 0.000 & 0.000 & 0.000 & 0.000 & 0.000 & 0.000 \\
\hline
\end{tabular}

Fuente: elaboración propia a partir de las regresiones cuantílicas. (Ver anexo).

Tabla 10. Diferencias intercuantílicas educación adultos

\begin{tabular}{|l|r|r|r|r|r|r|}
\hline & \multicolumn{1}{|c|}{$\mathbf{2 5 - 1}$} & $\mathbf{5 - 2 5}$ & \multicolumn{1}{|c|}{$\mathbf{9 - 1}$} & $\mathbf{7 5 - 2 5}$ & $\mathbf{9 - 7 5}$ & \multicolumn{1}{c|}{$\mathbf{9 - 5}$} \\
\hline \multirow{2}{*}{ esc } & $-0,008235$ & $-0,0095484$ & $-0,0047817$ & $-0,0033335$ & 0,0067867 & 0,0130017 \\
\cline { 2 - 7 } & 0.050 & 0.015 & 0.091 & 0.299 & 0.002 & 0.000 \\
\hline \multirow{3}{*}{ constante } & 0,9275735 & 0,5829908 & 2,381469 & 0,9401358 & 0,5137595 & 0,8709045 \\
\cline { 2 - 7 } & 0.000 & 0.000 & 0.000 & 0.000 & 0.000 & 0.000 \\
\hline
\end{tabular}

Fuente: elaboración propia a partir de resultados del modelo. 
En las tablas 11 y 12 se ven los resultados de la experiencia para jóvenes y adultos. En ellas se observa que para los jóvenes la experiencia explica la diferencia entre todos los cuantiles para los dos grupos, esto significa que en los extremos (9010), entre los más altos (90-75), entre los más altos y los medios (90-50) y (75-25) y entre los medios y bajos y (50-25) y la parte inferior (25-10). Esto quiere decir que el tener más experiencia explica la diferencia salarial entre cuantiles, la experiencia explica mejor las diferencias de los salarios en la distribución para llegar a los ingresos más altos. Para ambos grupos es significativo ver las diferencias entre los cuantiles en general.

Tabla 11. Diferencias intercuantílicas experiencia. Jóvenes.

\begin{tabular}{|l|r|r|r|r|r|r|}
\hline & \multicolumn{1}{|c|}{$\mathbf{2 5 - 1}$} & $\mathbf{5 - 2 5}$ & $\mathbf{9 - 1}$ & $\mathbf{7 5 - 2 5}$ & $\mathbf{9 - 7 5}$ & \multicolumn{1}{c|}{$\mathbf{9 - 5}$} \\
\hline \multirow{2}{*}{ expe } & $-0,0006321$ & 0,0002728 & 0,0011365 & 0,0013097 & 0,000459 & 0,0014958 \\
\cline { 2 - 7 } & 0.000 & 0.000 & 0.000 & 0.000 & 0.000 & 0.000 \\
\hline \multirow{2}{*}{ constante } & 0,9207152 & 0,4729587 & 2,177032 & 0,7716902 & 0,4846266 & 0,7833581 \\
\cline { 2 - 7 } & 0.000 & 0.000 & 0.000 & 0.000 & 0.000 & 0.000 \\
\hline
\end{tabular}

Fuente: elaboración propia a partir de resultados del modelo.

Tabla 12. Diferencias intercuantílicas experiencia. Adultos.

\begin{tabular}{|l|r|r|r|r|r|r|}
\hline & \multicolumn{1}{|c|}{$\mathbf{2 5 - 1}$} & $\mathbf{5 - 2 5}$ & \multicolumn{1}{|c|}{$\mathbf{9 - 1}$} & $\mathbf{7 5 - 2 5}$ & $\mathbf{9 - 7 5}$ & \multicolumn{1}{c|}{$\mathbf{9 - 5}$} \\
\hline \multirow{2}{*}{ expe } & $-0,0005508$ & $-0,0005508$ & 0,0012154 & 0,0012055 & 0,0005606 & 0,0016602 \\
\cline { 2 - 7 } & 0.000 & 0.000 & 0.000 & 0.000 & 0.000 & 0.000 \\
\hline \multirow{2}{*}{ constante } & 0,9427288 & 0,9427288 & 2,173451 & 0,765836 & 0,4648865 & 0,7556191 \\
\cline { 2 - 7 } & 0.000 & 0.000 & 0.000 & 0.000 & 0.000 & 0.000 \\
\hline
\end{tabular}

Fuente: elaboración propia a partir de resultados del modelo.

Por último, se comprobó si había diferencias significativas entre los retornos de la educación y la experiencia en la muestra en general y en cada grupo. La prueba de significancia que se usó es la de Wilcoxon (1945), en donde se compara la mediana de la educación y la experiencia. De este modo se planteó la hipótesis de igualdad entre ambas medianas de las variables $(\mathrm{H} 0$ : esc $=$ expe $)$ para toda la muestra y para cada grupo. En definitiva, no se aceptó la hipótesis nula en la muestra general y en cada grupo. Por tanto, hay diferencias estadísticamente significativas entre estas variables.

\section{Resultados por departamentos y sectores}

Acontinuación se muestran los resultados de las estimaciones de los departamentos donde trabajan y la rama de actividad económica. Estas variables se evaluaron en el segundo grupo de ecuaciones, por medio de las regresiones cuantílicas. 
Se muestran los resultados aparte para facilitar el análisis y para enfatizar en qué zonas del país y en qué sectores de la economía se dan mayores salarios. En las cuatro regresiones se observaron comportamientos muy similares para estas dos variables, por tanto los resultados que se mostrarán serán por cuantiles, que es donde se dan las diferencias, para jóvenes y adultos.

Como se puede ver en las tablas 9 y 10 , para los jóvenes las regiones que más influyen en el salario de forma positiva SOn BOGOTÁ, CESAR, CÓRDOBA, META y SANTANDER en los cuartiles 1025 y 50, y las zonas que afectan en mayor medida el salario de forma negativa son BOYACÁ, CALDAS, CAUCA, CHOCÓ y HUILA. Para los adultos, los mayores efectos positivos se dieron en BOGOTÁ, META y VICHADA para los cuantiles 10, 25, 50 y 75 . Los mayores efectos negativos se vieron en ATLÁNTICO, BOYACÁ, CALDAS, CAUCA, CHOCÓ, GUAJIRA, NARIÑO y ARAUCA. Por tanto, como era de esperarse, BOGOTÁ es la ciudad que mayores retornos salariales presenta, por ser la capital y la región que más mano de obra demanda. Las regiones que mayor efecto negativo presentan son BOYACÁ, CALDAS, CAUCA y CHOCÓ, tanto para jóvenes como para adultos; es decir, son las regiones que por el hecho de trabajar en ellas el salario disminuye. 
Tabla 13. Resultados zona geográfica para jóvenes 2015

\begin{tabular}{|c|c|c|c|c|c|}
\hline & cuartil 10 & cuartil 25 & cuartil 50 & cuartil 75 & cuartil 90 \\
\hline \multirow{2}{*}{$\begin{array}{l}\text { Antioquia } \\
\text { Atlántico }\end{array}$} & omitido & omitido & omitido & omitido & omitido \\
\hline & efecto positivo & no significativo & efecto negativo & efecto negativo & efecto negativo \\
\hline \multirow{2}{*}{$\begin{array}{l}\text { Bogotá } \\
\text { Bolívar }\end{array}$} & efecto positivo & efecto positivo & efecto positivo & no significativo & no significativo \\
\hline & no significativo & no significativo & efecto negativo & efecto negativo & efecto negativo \\
\hline \multirow{2}{*}{$\begin{array}{l}\text { Boyacá } \\
\text { Caldas }\end{array}$} & no significativo & efecto negativo & efecto negativo & efecto negativo & efecto negativo \\
\hline & no significativo & efecto negativo & efecto negativo & efecto negativo & efecto negativo \\
\hline \multirow{2}{*}{$\begin{array}{l}\text { Caquetá } \\
\text { Cauca }\end{array}$} & no significativo & no significativo & efecto negativo & efecto negativo & efecto negativo \\
\hline & efecto positivo & efecto negativo & efecto negativo & efecto negativo & efecto negativo \\
\hline \multirow{2}{*}{$\begin{array}{l}\text { Cesar } \\
\text { Córdoba }\end{array}$} & efecto positivo & efecto positivo & efecto negativo & efecto negativo & efecto negativo \\
\hline & efecto positivo & efecto positivo & efecto negativo & efecto negativo & efecto negativo \\
\hline Cundinamarca & no significativo & no significativo & efecto negativo & efecto negativo & efecto negativo \\
\hline Chocó & no significativo & efecto negativo & efecto negativo & efecto negativo & efecto negativo \\
\hline Huila & no significativo & efecto negativo & efecto negativo & efecto negativo & efecto negativo \\
\hline Guajira & no significativo & no significativo & efecto negativo & efecto negativo & efecto negativo \\
\hline Magdalena & efecto positivo & no significativo & efecto negativo & efecto negativo & efecto negativo \\
\hline Meta & efecto positivo & efecto positivo & efecto positivo & no significativo & efecto negativo \\
\hline Nariño & no significativo & no significativo & efecto negativo & efecto negativo & efecto negativo \\
\hline Norte de Santander & efecto positivo & no significativo & efecto negativo & efecto negativo & efecto negativo \\
\hline Quindío & efecto positivo & no significativo & efecto negativo & efecto negativo & efecto negativo \\
\hline Risaralda & efecto positivo & no significativo & efecto negativo & efecto negativo & efecto negativo \\
\hline Santander & efecto positivo & efecto positivo & efecto positivo & no significativo & no significativo \\
\hline Sucre & efecto positivo & no significativo & efecto negativo & efecto negativo & efecto negativo \\
\hline Tolima & no significativo & no significativo & efecto negativo & efecto negativo & efecto negativo \\
\hline Valle del Cauca & efecto positivo & no significativo & efecto negativo & efecto negativo & efecto negativo \\
\hline Arauca & no significativo & no significativo & efecto negativo & efecto negativo & efecto negativo \\
\hline Casanare & no significativo & efecto positivo & efecto negativo & no significativo & no significativo \\
\hline Putumayo & no significativo & efecto positivo & efecto negativo & efecto negativo & no significativo \\
\hline San Andrés & omitido & omitido & omitido & omitido & omitido \\
\hline Vichada & no significativo & no significativo & efecto negativo & efecto negativo & efecto negativo \\
\hline
\end{tabular}

Fuente: elaboración propia a partir de las regresiones cuantílicas. (Ver anexo). 
Retornos salariales para Colombia, un análisis cuantílico

Cristian Darío Castillo Robayo - Julimar Da Silva Bichara - Manuel Pérez Trujillo

Tabla 14. Resultados zona geográfica para adultos 2015.

\begin{tabular}{|c|c|c|c|c|c|}
\hline & cuartil 10 & cuartil 25 & cuartil 50 & cuartil 75 & cuartil 90 \\
\hline \multirow{2}{*}{$\begin{array}{l}\text { Antioquia } \\
\text { Atlántico }\end{array}$} & omitido & omitido & omitido & omitido & omitido \\
\hline & efecto positivo & efecto negativo & efecto negativo & efecto negativo & efecto negativo \\
\hline \multirow{2}{*}{$\begin{array}{l}\text { Bogotá } \\
\text { Bolívar }\end{array}$} & efecto positivo & efecto positivo & efecto positivo & efecto positivo & no significativo \\
\hline & efecto positivo & no significativo & efecto negativo & efecto negativo & efecto negativo \\
\hline \multirow{2}{*}{$\begin{array}{l}\text { Boyacá } \\
\text { Caldas }\end{array}$} & no significativo & efecto negativo & efecto negativo & efecto negativo & efecto negativo \\
\hline & efecto positivo & efecto negativo & efecto negativo & efecto negativo & efecto negativo \\
\hline \multirow{2}{*}{$\begin{array}{l}\text { Caquetá } \\
\text { Cauca }\end{array}$} & efecto positivo & no significativo & efecto negativo & efecto negativo & efecto negativo \\
\hline & no significativo & efecto negativo & efecto negativo & efecto negativo & efecto negativo \\
\hline \multirow{2}{*}{$\begin{array}{l}\text { Cesar } \\
\text { Córdoba } \\
\end{array}$} & efecto positivo & efecto positivo & efecto negativo & efecto negativo & efecto negativo \\
\hline & efecto positivo & efecto positivo & efecto negativo & efecto negativo & efecto negativo \\
\hline Cundinamarca & no significativo & no significativo & no significativo & efecto negativo & efecto negativo \\
\hline Chocó & efecto positivo & efecto negativo & efecto negativo & efecto negativo & efecto negativo \\
\hline Huila & efecto positivo & no significativo & efecto negativo & efecto negativo & efecto negativo \\
\hline Guajira & no significativo & efecto negativo & efecto negativo & efecto negativo & efecto negativo \\
\hline Magdalena & efecto positivo & no significativo & efecto negativo & efecto negativo & efecto negativo \\
\hline Meta & efecto positivo & efecto positivo & efecto positivo & no significativo & efecto negativo \\
\hline Nariño & no significativo & efecto negativo & efecto negativo & efecto negativo & efecto negativo \\
\hline Norte de Santander & efecto positivo & no significativo & efecto negativo & efecto negativo & efecto negativo \\
\hline Quindío & no significativo & no significativo & efecto negativo & efecto negativo & efecto negativo \\
\hline Risaralda & efecto positivo & efecto positivo & no significativo & efecto negativo & efecto negativo \\
\hline Santander & efecto positivo & efecto positivo & no significativo & efecto positivo & no significativo \\
\hline Sucre & efecto positivo & no significativo & efecto negativo & efecto negativo & efecto negativo \\
\hline Tolima & no significativo & no significativo & efecto negativo & efecto negativo & efecto negativo \\
\hline Valle del Cauca & no significativo & no significativo & efecto negativo & efecto negativo & efecto negativo \\
\hline Arauca & efecto positivo & efecto negativo & efecto negativo & efecto negativo & efecto negativo \\
\hline Casanare & no significativo & no significativo & no significativo & no significativo & efecto negativo \\
\hline Putumayo & no significativo & efecto positivo & no significativo & no significativo & efecto negativo \\
\hline San Andrés & omitido & omitido & omitido & omitido & omitido \\
\hline Vichada & efecto positivo & efecto negativo & no significativo & efecto positivo & efecto negativo \\
\hline
\end{tabular}

Fuente: elaboración propia a partir de las regresiones cuantílicas. (Ver anexo).

Explotación de minas y canteras y la rama de organizaciones y órganos extraterritoriales fueron los que mayores retornos mostraron en general tanto para jóvenes como para adultos. Para los jóvenes, en los cuantiles 2550 y 75 todos los sectores mostraron efectos positivos; en el cuartil 90, que representa los mayores salarios de la distribución, los que mostraron efecto positivo fueron: suministro de electricidad, gas y agua, construcción, hoteles y restaurantes, transporte, almacenamiento y comunicaciones, administración pública y defensa; planes de seguridad social de afiliación obligatoria, enseñanza, servicios sociales y de salud y organizaciones y órganos extraterritoriales.

En los adultos, la rama de actividad pierde significancia y son pocos los sectores que muestran efectos significativos y positivos, entre ellos industria manufacturera en el cuantil 50 y 75 y organizaciones y órganos extraterritoriales en el cuartil 50 y 90; y explotación de minas y canteras en el cuantil 75 y 90. 
Tabla 15. Resultados rama de actividad económica para jóvenes 2015

\begin{tabular}{|c|c|c|c|c|c|}
\hline & cuartil 10 & cuartil 25 & cuartil 50 & cuartil 75 & cuartil 90 \\
\hline $\begin{array}{l}\text { Agricultura, ganade- } \\
\text { ría, caza y silvicultura }\end{array}$ & no significativo & efecto positivo & efecto positivo & efecto positivo & no significativo \\
\hline Pesca & no significativo & efecto positivo & efecto positivo & efecto positivo & no significativo \\
\hline $\begin{array}{l}\text { Explotación de minas } \\
\text { y canteras }\end{array}$ & no significativo & efecto positivo & efecto positivo & efecto positivo & no significativo \\
\hline $\begin{array}{l}\text { Industrias manufac- } \\
\text { tureras }\end{array}$ & no significativo & efecto positivo & efecto positivo & efecto positivo & no significativo \\
\hline $\begin{array}{l}\text { Suministro de electri- } \\
\text { cidad, gas y agua }\end{array}$ & no significativo & efecto positivo & efecto positivo & efecto positivo & efecto positivo \\
\hline Construcción & no significativo & efecto positivo & efecto positivo & efecto positivo & efecto positivo \\
\hline $\begin{array}{l}\text { Comercio al por mayor } \\
\text { y al por menor; repara- } \\
\text { ción de vehículos auto- } \\
\text { motores, motocicletas, } \\
\text { efectos personales y } \\
\text { enseres domésticos }\end{array}$ & no significativo & efecto positivo & efecto positivo & efecto positivo & efecto positivo \\
\hline Hoteles y restaurantes & no significa tivo & efecto positivo & efecto positivo & efecto positivo & efecto positivo \\
\hline $\begin{array}{l}\text { Transporte, almacena- } \\
\text { miento y comunicacio- } \\
\text { nes }\end{array}$ & no significativo & efecto positivo & efecto positivo & efecto positivo & efecto positivo \\
\hline $\begin{array}{l}\text { Intermediación finan- } \\
\text { ciera }\end{array}$ & no significativo & efecto positivo & efecto positivo & efecto positivo & no significativo \\
\hline $\begin{array}{l}\text { Actividades inmobilia- } \\
\text { rias, empresariales y } \\
\text { de alquiler }\end{array}$ & no significativo & efecto positivo & efecto positivo & efecto positivo & no significativo \\
\hline $\begin{array}{l}\text { Administración pública } \\
\text { y defensa; planes de se- } \\
\text { guridad social de afilia- } \\
\text { ción obligatoria }\end{array}$ & no significativo & efecto positivo & efecto positivo & efecto positivo & efecto positivo \\
\hline Enseñanza & no significativo & efecto positivo & efecto positivo & efecto positivo & efecto positivo \\
\hline $\begin{array}{l}\text { Servicios sociales y } \\
\text { de salud }\end{array}$ & no significativo & efecto positivo & efecto positivo & efecto positivo & efecto positivo \\
\hline $\begin{array}{l}\text { Otras actividades de } \\
\text { servicios comunitarios, } \\
\text { sociales y personales }\end{array}$ & no significativo & efecto positivo & efecto positivo & efecto positivo & no significativo \\
\hline $\begin{array}{l}\text { Actividades de hoga- } \\
\text { res privados como } \\
\text { empleadores y activi- } \\
\text { dades no diferenciadas } \\
\text { de hogares privados } \\
\text { como productores }\end{array}$ & no significativo & efecto positivo & efecto positivo & efecto positivo & no significativo \\
\hline $\begin{array}{l}\text { Organizaciones y } \\
\text { órganos } \\
\text { extraterritoriales }\end{array}$ & no significativo & efecto positivo & efecto positivo & efecto positivo & no significativo \\
\hline
\end{tabular}

Fuente: elaboración propia a partir de las regresiones cuantílicas. (Ver anexo). 
Retornos salariales para Colombia, un análisis cuantílico

Cristian Darío Castillo Robayo - Julimar Da Silva Bichara - Manuel Pérez Trujillo

Tabla 16. Resultados rama de actividad económica para adultos 2015

\begin{tabular}{|c|c|c|c|c|c|}
\hline & cuartil 10 & cuartil 25 & cuartil 50 & cuartil 75 & cuartil 90 \\
\hline $\begin{array}{l}\text { Agricultura, ganadería, } \\
\text { caza y silvicultura }\end{array}$ & efecto negativo & no significativo & no significativo & efecto negativo & no significativo \\
\hline Pesca & efecto negativo & no significa tivo & no significa tivo & efecto negativo & no significativo \\
\hline $\begin{array}{l}\text { Explotación de minas } \\
\text { y canteras }\end{array}$ & efecto negativo & no significativo & no significativo & efecto positivo & efecto positivo \\
\hline $\begin{array}{l}\text { Industrias manufactu- } \\
\text { reras }\end{array}$ & efecto negativo & no significativo & efecto positivo & efecto negativo & no significativo \\
\hline $\begin{array}{l}\text { Suministro de electrici- } \\
\text { dad, gas y agua }\end{array}$ & efecto negativo & no significativo & no significa tivo & no significativo & no significa tivo \\
\hline Construcción & efecto negativo & no significativo & no significativo & no significativo & no significativo \\
\hline $\begin{array}{l}\text { Comercio al por mayor } \\
\text { y al por menor; repara- } \\
\text { ción de vehículos auto- } \\
\text { motores, motocicletas, } \\
\text { efectos personales y } \\
\text { enseres domésticos } \\
\end{array}$ & efecto negativo & no significativo & no significativo & no significativo & no significativo \\
\hline Hoteles y restaurantes & efecto negativo & no significativo & no significativo & no significativo & no significativo \\
\hline $\begin{array}{l}\text { Transporte, almacena- } \\
\text { miento y comunicacio- } \\
\text { nes }\end{array}$ & efecto negativo & no significativo & no significativo & efecto positivo & no significativo \\
\hline $\begin{array}{l}\text { Intermediación finan- } \\
\text { ciera }\end{array}$ & efecto negativo & no significativo & no significativo & efecto positivo & no significativo \\
\hline $\begin{array}{l}\text { Actividades inmobilia- } \\
\text { rias, empresariales y de } \\
\text { alquiler }\end{array}$ & efecto negativo & no significativo & no significativo & efecto positivo & no significativo \\
\hline $\begin{array}{l}\text { Administración pública } \\
\text { y defensa; planes de se- } \\
\text { guridad social de afilia- } \\
\text { ción obligatoria }\end{array}$ & efecto negativo & no significativo & no significa tivo & efecto positivo & no significativo \\
\hline Enseñanza & efecto negativo & no significativo & no significativo & no significativo & no significativo \\
\hline $\begin{array}{l}\text { Servicios sociales y } \\
\text { de salud }\end{array}$ & efecto negativo & no significativo & no significativo & no significativo & no significativo \\
\hline $\begin{array}{l}\text { Otras actividades de } \\
\text { servicios comunitarios, } \\
\text { soefecto negativo } \\
\text { ciales y personales } \\
\end{array}$ & no significativo & no significativo & no significa tivo & no significativo & \\
\hline $\begin{array}{l}\text { Actividades de hogares } \\
\text { privados como emplea- } \\
\text { dores y actividades no } \\
\text { diferenciadas de hoga- } \\
\text { res privados como } \\
\text { productores }\end{array}$ & efecto negativo & no significativo & efecto negativo & efecto negativo & no significativo \\
\hline $\begin{array}{l}\text { Organizaciones } \\
\text { y órganos } \\
\text { extraterritoriales }\end{array}$ & efecto negativo & no significativo & efecto positivo & no significativo & efecto positivo \\
\hline
\end{tabular}

Fuente: elaboración propia a partir de las regresiones cuantílicas. (Ver anexo). 
Los resultados mostrados concuerdan con otros estudios sobre el tema. Para Psacharoupoulos (1994), los retornos de la educación han disminuido en varias regiones del mundo, y afirma, como se hizo en este estudio, que la educación también presenta rendimientos decrecientes. En Colombia también se han encontrado resultados similares, pero en estudios con otras metodologías, en cuanto a la disminución de los retornos de la educación. Ejemplo de ello está en Tenjo (1993), Castellar y Uribe (2003), Mora (2003), Prada (2006), y Uribe, Ortiz y García (2006).

Por último, el estudio referenciado de Zárate (2003) encontró que los retornos de la educación para el periodo 1991 al 2000, son altos en los cuantiles más altos, y los retornos de la experiencia son más altos para los nuevos trabajadores en los cuantiles bajos. Es decir, para los jóvenes representa un mayor retorno la experiencia. Esto confirma los resultados mostrados en cuanto los retornos de la experiencia son mayores para los jóvenes, pero no solo de cuantíles bajos, sino también de los altos.

\section{CONCLUSIONES}

La relación entre educación e ingresos laborales se ha estudiado ampliamente en la literatura económica, desde que se comenzó a creer, a mediados del siglo $\mathrm{XX}$, que era una variable esencial para el desarrollo de los países. Por su parte, la experiencia se toma en cuenta, pero sus beneficios en los retornos sobre los ingresos no se estudiaban tan a fondo. En la teoría del capital humano se habla de estos dos aspectos, no obstante, en los estudios se enfatiza más en los beneficios de la educación. En las teorías que critican el capital humano, se enfatiza más sobre los mayores beneficios de la experiencia sobre la productividad y los salarios.

En Colombia se han hecho varios esfuerzos por aumentar el capital humano, se han implementado políticas públicas en pro de generar acceso universal a la educación primaria y secundaria y fomentar la educación técnica y universitaria, sin embargo no se ha hecho tanto por facilitar la obtención de experiencia, en su mayor medida para los jóvenes, lo que provoca baja inserción laboral y bajos salarios. Esto es un problema, porque en la economía actual la experiencia genera más retornos que la educación, y hay muchos trabajadores con cualificaciones altas, pero con salarios por debajo de dichas cualificaciones.

La forma como tradicionalmente se han testeado los retornos del capital humano se basan en las ecuaciones de Mincer, en este caso se usaron de base estos modelos, pero se hicieron estimaciones por regresiones cuantílicas, para ver de mejor manera el comportamiento en toda la distribución del ingreso para jóvenes y adultos. Esto confirmó la hipótesis de que la experiencia en la actualidad genera mayores retornos sobre el ingreso, por encima de la educación, para los jóvenes. 
Así mismo, se encontró que la explotación de minas y canteras y la rama de organizaciones y órganos extraterritoriales fueron las que mayores retornos salariales mostraron en general tanto para jóvenes como para adultos. Además, BOGOTÁ es la ciudad que mayores retornos salariales presenta, por ser la capital y la región que más mano de obra demanda; y las regiones que impactan de forma negativa fueron BOYACÁ, CALDAS, CAUCA y CHOCÓ, tanto para jóvenes como para adultos; es decir, son las regiones donde por el hecho de trabajar en ellas el salario es más bajo.

Eso refleja que en el país los mayores salarios se dan en actividades que requieren menos cantidad de trabajadores y cualificaciones no tan altas en la mayoría de ellos. Las regiones que presentan resultados negativos son en su mayoría consideradas zonas agrarias, esto dejaría ver las dificultades de obtener salarios altos trabajando en la agricultura en Colombia.

Por tanto, se deben diseñar más políticas en pro de complementar la formación de los jóvenes mediante el acceso a más oportunidades para adquirir experiencia, que les permita encontrar más rápido un empleo y mejor remunerado, lo cual se puede lograr implementando más cursos de formación asociados a los requerimientos de quienes demandan empleo, para que se relacione la educación con la actividad profesional.

\section{REFERENCIAS}

Arrow, K. J. (1973). Higher Education as a Filter. Journal of Public Economics, 2(3), 193216.

Azariadis, C. \& Drazen, A. (1990). Threshold Externalities in Economic Development. The Quarterly Journal of Economics, 501-526.

Azariadis, C. \& Stiglitz, J. E. (1983). Implicit Contracts and Fixed Price Equilibria. The Quarterly Journal of Economics, 2-22.

Barro, R. J. \& Lee, J. W. (2001). International Data on Educational Attainment: Updates and Implications. Oxford Economic Papers, 53(3), 541-563.

Becker, G. S. (1964a). Human Capital: A Theoretical and Empirical Analysis, with Special Reference to Education. Illinois, USA: University of Illinois at UrbanaChampaign's Academy for Entrepreneurial Leadership Historical Research Reference in Entrepreneurship. 
Becker, G. S. (1964 b). Human Capital Theory. New York: Columbia.

Benito, S. M. R., Trujillo, M. P., Ortiz, L. P., Lasierra, J. M., \& da Silva Bichara, J. (2014). Economía del trabajo y política laboral. Madrid: Pirámide.

Berger, S. \& Piore, M. J. (1980). Dualism and Discontinuity in Industrial Societies. Cambridge, MA: Cambridge University Press.

Blackburn, M. L. \& Neumark, D. (1991). Omitted-ability bias and the Increase in the Return to Schooling (No. w3693). Cambridge, MA: National Bureau of Economic Research.

Blanchflower, D. G. \& Oswald, A. J. (1995). An Introduction to the Wage Curve. The Journal of Economic Perspectives, 153-167.

Boissiere, M., Knight, J. B. \& Sabot, R. H. (1985). Earnings, schooling, ability, and cognitive skills. The American Economic Review, 75(5), 1016-1030.

Brown, C., \& Medoff, J. L. (1989). The Employer Size-Wage Effect (No. w2870). Cambridge, MA: National Bureau of Economic Research.

Buchinsky, M. (1994). Changes in the US Wage Structure 1963-1987: Application of Quantile Regression. Econometrica: Journal of the Econometric Society, 405-458.

Buchinsky, M. (1998). Recent Advances in Quantile Regression Models: A Practical Guideline for Empirical Research. Journal of Human Resources, 88-126.

Cahuc, P., Carcillo, S., Zylberberg, A. \& McCuaig, W. (2014). Labor Economics. Cambridge, MA: MIT Press.

Castellar, C. \& Uribe, J. (2003). La tasa de retorno de la educación: teoría y evidencia micro y macroeconómicas en el área metropolitana de Cali. Documento de Trabajo, (3090).

Collins, R. (1986). Las teorías técnico-funcionalista y credencialista de la estratificación educativa. Revista Educación y Sociedad, (5).

Doeringer, P. B. \& Piore, M. J. (1983). El paro y el mercado dual de trabajo. En L. Toharia (comp.) El mercado de trabajo: teorías y aplicaciones (pp. 341-388). Madrid: Alianza.

Fitzenberger, B., Hujer, R., MaCurdy, \& T. Schnabel, R. (2001). Las pruebas de las tendencias salariales uniformes en Alemania Occidental: un análisis de cohortes 
utilizando regresiones por cuantíles para datos censurados. Empírica Economía, 26, 41-86.

Flórez, A. La desigualdad salarial de género medida por regresión cuantílica: el impacto del capital humano, cultural y social. Revista Mexicana de Ciencias Políticas y Sociales, 60(223), 287-315.

Freitas, A. A. (2015). La desigualdad salarial de género medida por regresión cuantílica: el impacto del capital humano, cultural y social. Revista Mexicana de Ciencias Políticas y Sociales, 60(223), 287-315.

Galvis, L. A. (2010). Diferenciales salariales por género y región en Colombia: una aproximación con regresión por cuantiles. Revista de Economía del Rosario, 13(2).

Katz, D. \& Kearney, M. (2004). US Wage and Consumption Inequality Trends: A Reassessment of Basic Facts and Alternative Explanations. Working Paper. Cambridge, MA: Massachusetts Institute of Technology.

Kerr, C. (1985). Mercados de trabajo y determinación de los salarios: la “balcanización” de los mercados de trabajo y otros ensayos. Madrid: Ministerio de Trabajo y Seguridad Social.

Keynes, J. M. (2007). The General Theory of Employment, Interest and Money. New Delhi, India: Atlantic Publishers \& Dist.

Knight, J. B. (1979). Job Competition, Occupational Production Functions, and Filtering Down. Oxford Economic Papers, 31(2), 187-204.

Koenker, R. \& Bassett Jr, G. (1978). Regression quantiles. Econometrica: Journal of the Econometric Society, 33-50.

López, H. A. \& Mora, H. M. (2007). Cálculo de los estimadores de regresión cuantílica lineal por medio del método ACCPM. Revista Colombiana de Estadística, 30(1), 53-68.

Lucas, R. E. (1988). On the Mechanics of Economic Development. Journal of Monetary Economics, 22(1), 3-42.

Malcomson, J. M. (1981). Unemployment and the Efficiency Wage Hypothesis. The Economic Journal, 848-866.

Mankiw, N. G., Romer, D. \& Weil, D. N. (1990). A Contribution to the Empirics of Economic Growth (No. w3541). Cambridge, MA: National Bureau of Economic Research. 
Mincer, J. (1974). Schooling, Experience and Earnings. Human Behavior \& Social Institutions, (2).

Mora, J. J. (2003). Sheepskin effects and screening in Colombia. Colombian Economic Journal, 1(1), 95-108.

Mushkin, S. J. (1962). Economics of Higher Education. USA: US DHEW.

Nielsen, H. S. \& Rosholm, M. (2001). The Public-Private Sector Wage Gap in Zambia in the 1990s: A Quantile Regression Approach. In Economic Applications of Quantile Regression (pp. 169-182). Heidelberg, Germany: Physica-Verlag HD.

Piore, M. J. (1983). Labor Market Segmentation: To What Paradigm does it belong? The American Economic Review, 249-253.

Prada, C. F. (2006). ¿Es rentable la decisión de estudiar en Colombia? Ensayos sobre Política Económica, 24(51), 226-323.

Psacharopoulos, G. (1994). Returns to Investment in Education: A Global Update. World Development, 22(9), 1325-1343.

Ruesga, S., Pérez, L., Pérez, M., Lasierra, J. \& Bichara, J. (2014). Economía del trabajo y política laboral. Madrid: Pirámide.

Schmid, G. (2008). Full Employment in Europe: Managing Labour Market Transitions and Risks. Northampton, MA: Edward Elgar Publishing.

Schultz, T. W. (1961). Investment in human capital. The American economic review, $1-17$.

Selowsky, M. (1968). The Effect of Unemployment Labour-Market Segmentation and the Returns to Educated: The Case of Colombia. Boston, MA: Harvard University Centre for International Affairs.

Solow, R. M. (1956). A Contribution to the Theory of Economic Growth. The Quarterly Journal of Economics, 65-94.

Spence, M. (1973). Job Market Signaling. The Quarterly Journal of Economics, 355374.

Stanford University, Institute for Mathematical Studies in the Social Sciences \& Stiglitz, J. E. (1975). Information and Economic Analysis. Stanford, CA: Stanford University. 
Stiglitz, J. E. (1984). Equilibrium wage distributions (No. w1337). Cambridge, MA: National Bureau of Economic Research.

Taubman, P. \& Wales, T. (1972). Mental Ability and Higher Educational Attainment in the 20th Century. New Jersey: McGrsw Hill.

Tenjo, J. (1993). Evolución de los retornos a la inversión en educación 1976-1989. Planeación y Desarrollo, 24(85), 102.

Thurow, L. (1983). Dangerous Currents: The State of Economics. Oxford, UK: Oxford University Press OUP Catalogue.

Uribe, J. I., Ortiz, C. H. \& García, G. A. (2006). La segmentación del mercado laboral colombiano en la década de los noventa. Bogotá: DNP.

Vargas, C. O. (2011). Desigualdad de salarios en Colombia: evidencia a partir de encuestas de hogares 1984-2010 (No. 661). Bogotá: Banco de la República de Colombia.

Vélez, F. A. (2011) Robustez a distribución para regresión cuantílica en datos longitudinales. Tesis para optar al título de magíster en estadística. Universidad Nacional de Colombia, Bogotá.

Wilcoxon, F. (1945). Individual Comparisons by Ranking Methods. Biometrics Bulletin, 1(6), 80-83.

Zárate, H. (2003). Cambios en la estructura salarial: una historia desde la regresión cuantílica. Borradores de Economía, Banco de la República, (245).

Zuluaga, B. \& Manzur, E. (2012). Notas de clase sobre el mercado laboral y las políticas de empleo (No. 010743). Cali, Colombia: Universidad ICESI. 